\title{
Physics Prospects with the JLab 12-GeV Upgrade
}

\author{
E.S. Smith*
}

Physics Division, Jefferson Lab, Newport News, Virginia, U.S.A.

Increasing the beam energy of the CEBAF accelerator to $12 \mathrm{GeV}$ will greatly expand the physics opportunities to explore the nature of strongly interacting matter. The primary thrusts of the experimental program will include the study of quark confinement and the direct exploration of the quark-gluon structure of hadrons and nuclei in the valence-quark region.

\section{INTRODUCTION}

There are important physics questions that cannot be addressed at the present time due to the lack of an electron accelerator with the combination of high energy, high duty-cycle, and the high beam power required to study rare phenomena. Of particular importance are the study of a) the origin of quark confinement by searching for $q \bar{q}$ systems with excited flux tubes, b) the longitudinal momentum distribution of the valence quarks in the nucleon, and c) quark-quark correlations via exclusive processes (by exploiting the recently developed formalism of the Generalized Parton Distributions). Another area of great interest, which is covered in another contribution to this conference, is the high- $Q^{2}$ behavior of elastic and transition form factors which are sensitive to the high-momentum components of the valence quark wavefunctions [1]. Therefore, plans have been developed, and endorsed by the nuclear physics community [2], for increasing the energy of the CEBAF accelerator to $12 \mathrm{GeV}$, and also upgrading the experimental equipment to match the higher energy [3-7].

The Southeastern Universities Research Association (SURA), a university consortium, operates the Thomas Jefferson National Accelerator Facility, also called Jefferson Lab (or JLab), for the U.S. Department of Energy. The main instrument is the Continuous Electron Beam Accelerator Facility, CEBAF, a superconducting electron accelerator capable of delivering three electron beams with a maximum beam power of $1 \mathrm{MW}$ and $100 \%$ duty-cycle for simultaneous electron scattering experiments in three experimental areas, Halls A, B, and C.

The halls contain complementary equipment to cover a wide range of physics problems. Hall A has two high resolution magnetic spectrometers for experiments that require the precise reconstruction of the mass of the unobserved hadronic final state. Hall B is equipped with a toroidal multi-gap spectrometer (CEBAF Large Acceptance Spectrometer, CLAS) for the detection of several, only loosely correlated particles in the hadronic

${ }^{*}$ This work is supported in part by the U.S. Department of Energy, including DOE Contract No. DEAC05-84ER40150. 
final state. The main instruments in Hall $\mathrm{C}$ are two focusing magnetic spectrometers: the High Momentum Spectrometer (HMS) and the Short Orbit Spectrometer (SOS).

\section{FACILITY UPGRADES}

The electron accelerator at Jefferson Lab consists of a pair of interconnected linacs, each comprising 20 cryomodules, with each cryomodule containing eight superconducting radiofrequency (SRF) accelerating cavities. On average, these cavities have exceeded their original design specification by $50 \%$. It is the success of this technology that has opened up the possibility of a simple upgrade for the current energy of $6 \mathrm{GeV}$ to $12 \mathrm{GeV}$. With expected further improvements in SRF technology and with the production of a new compact cryomodule based on seven-cell cavities, doubling the energy of the machine can be attained at modest cost. Straightforward modifications of the re-circulating arcs will accommodate the higher energies.

The highest energy of $12 \mathrm{GeV}$ will be available in a new experimental area at the end of the north linac, Hall D. This area will be devoted to the photoproduction of exotic mesons using a linearly polarized photon beam and the GlueX large-acceptance detector. The three existing areas will be able to use any multiple of $2.2 \mathrm{GeV}$ (or a lower energy), up to $11 \mathrm{GeV}$. Their present equipment will be upgraded to take advantage of the higher energy. In Hall A, a broad-range spectrometer (Medium Acceptance Spectrometer, MAD) will be added. The CLAS in Hall B will be upgraded to higher luminosity and improved forward coverage. In Hall C, the SOS will be replaced by a new Super High Momentum Spectrometer, SHMS, to cover small angles and high momenta.

\section{GLUONIC EXCITATIONS}

In the early 1970s, evidence that the masses of strongly interacting particles increased without limit as their internal angular momentum increased led the theorist Yoichiro Nambu to propose that the quarks inside these particles are "tied together" by strings. Lattice simulations of quantum chromodynamics (QCD) have demonstrated that Nambu's conjecture was essentially correct: in QCD, a string-like chromoelectric flux tube forms between distant static quarks, leading to their confinement with an energy proportional to the distance between them (see Fig. 1). The phenomenon of confinement is the most novel and spectacular prediction of QCD - unlike anything seen before.

The clear consequence of this picture, where mesons are composed of quarks connected by a flux tube, is the existence of families of mesons with an exited flux tube that exhibit gluonic degrees of freedom explicitly. Conventional mesons arise when the flux tube is in the ground state, as shown in Fig. 1 (right), and (hybrid) mesons correspond to states where the flux tube is excited. These lattice calculations were performed for heavy quarks, and models are required to extend this firm understanding to lighter quarks. But the most important properties of this system are determined by the model-independent features described above. The states are expected to have masses around $2 \mathrm{GeV}$, with a range of predicted mass as shown in Fig. 2. Furthermore, these excited states can have exotic quantum numbers which are missing from the $q \bar{q}$ spectrum because the flux-tube can independently posses angular momentum. Therefore mixing between exotic hybrids and $q \bar{q}$ states is not allowed, and searching for exotic quantum numbers provides a rich 

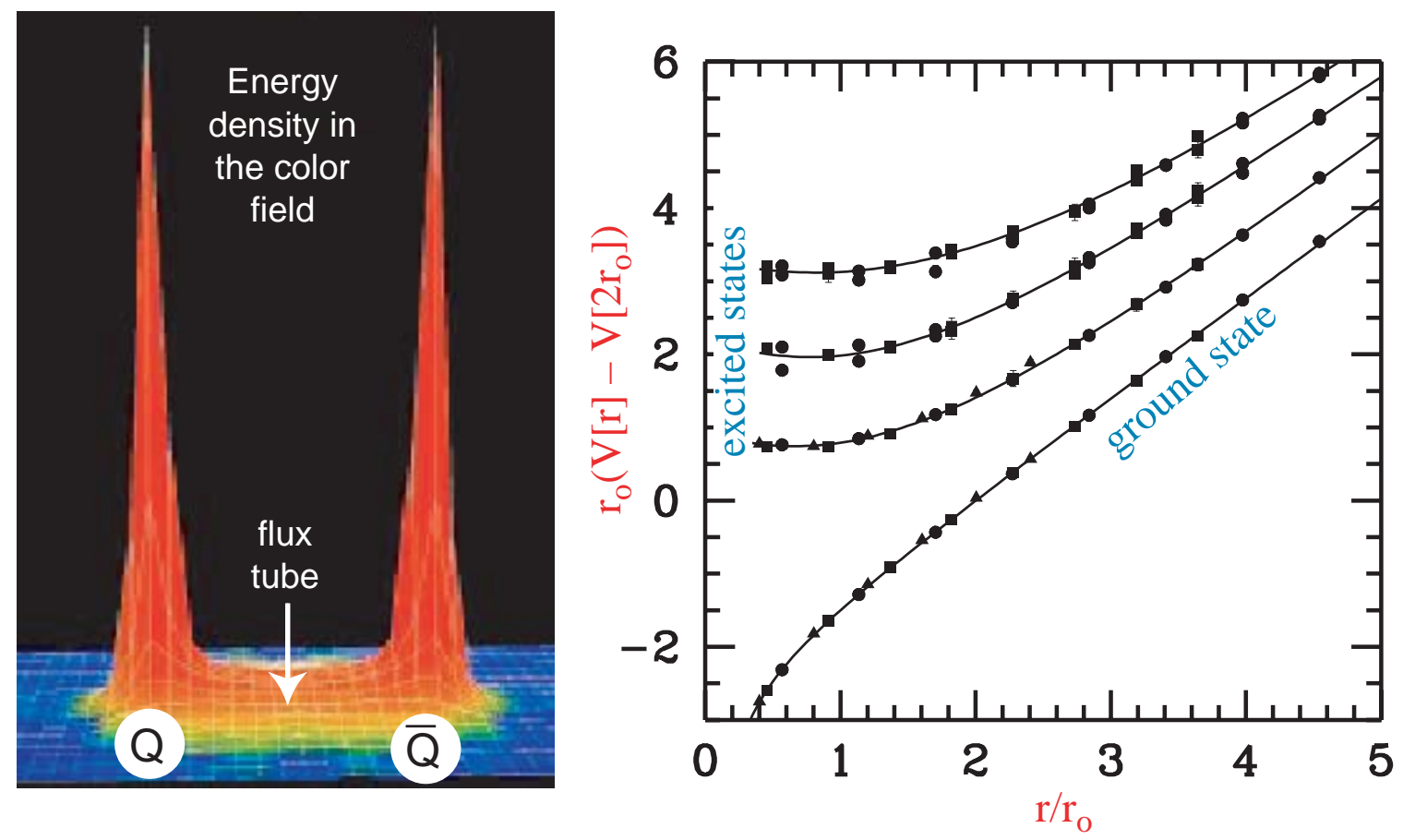

Figure 1. (left) A lattice QCD calculation of the energy density in the color field between a quark and an anti-quark. The density peaks at the positions of the quarks and is confined to a tube between the quarks. This calculation is for heavy quarks in the quenched approximation. (right) The corresponding potential between the quarks. The ground state potential has a $1 / r$ dependence at small distances and is linear for large distances.

area for experiments.

\subsection{Photo-production of Exotic Hybrids}

Photon beams are expected to be particularly favorable for the production of the exotic hybrids. The reason is that the photon sometimes behaves as a "virtual vector meson" with total quark spin $S=1$. When the flux tube in this $S=1$ system is excited, both ordinary and exotic $J^{P C}$ are possible. In contrast, when the spins are anti-parallel $(S=0)$, as in pion or kaon probes, the exotic combinations are not generated. To date, most meson spectroscopy has been done with incident pion, kaon, or proton probes, so it is not surprising that the experimental evidence to date for flux tube excitation is tentative. In contrast to hadron beams, high-flux photon beams of sufficient quality and energy to perform meson spectroscopy studies have not been available, so there are virtually no data on photoproduction of mesons with masses in the 1.5 to $3 \mathrm{GeV}$ region. Thus, experimenters have not been able to search for exotic hybrids precisely where they are expected to be found.

The optimal photon beam energy is set by several considerations. The interest lies in the mass range from $1.0 \mathrm{GeV} / \mathrm{c}^{2}$ up to about $2.7 \mathrm{GeV} / \mathrm{c}^{2}$, the region where light quark hybrids are expected to exist. Incident photon energies of $9 \mathrm{GeV}$ are sufficient to 


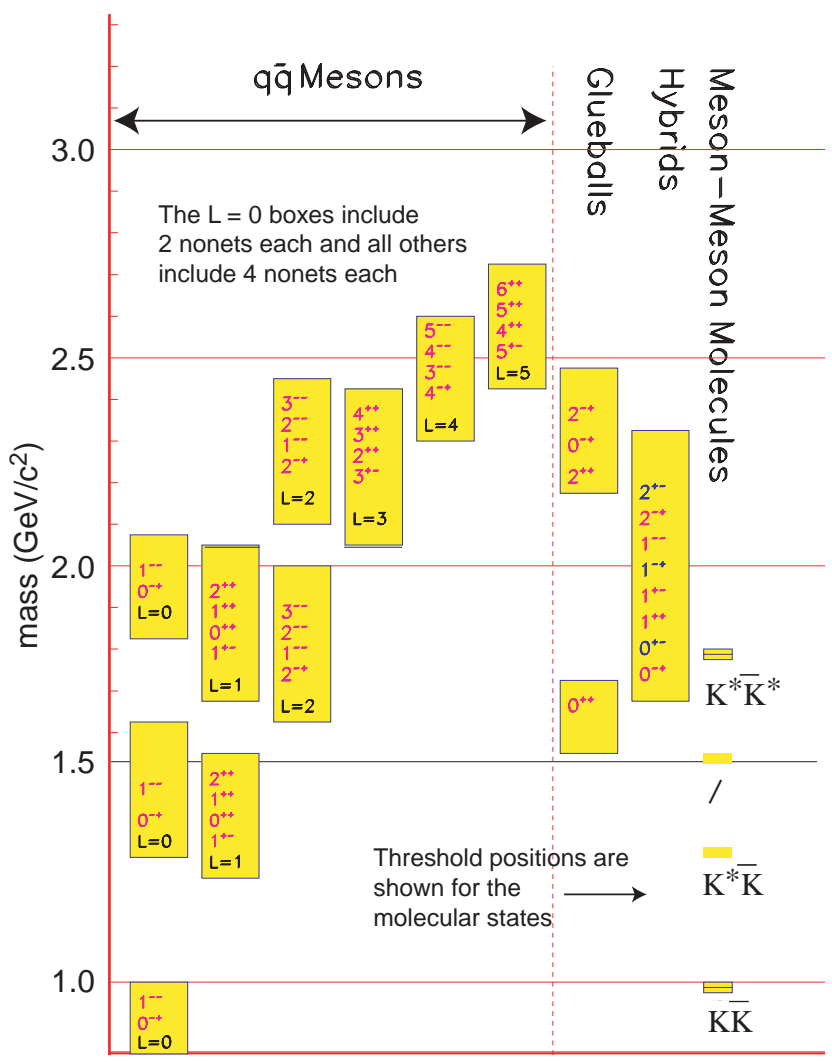

Figure 2. Excitation spectrum of the $q \bar{q}$ spectrum(left) and gluonic and molecular states (right). Exotic quantum numbers are indicated in blue.

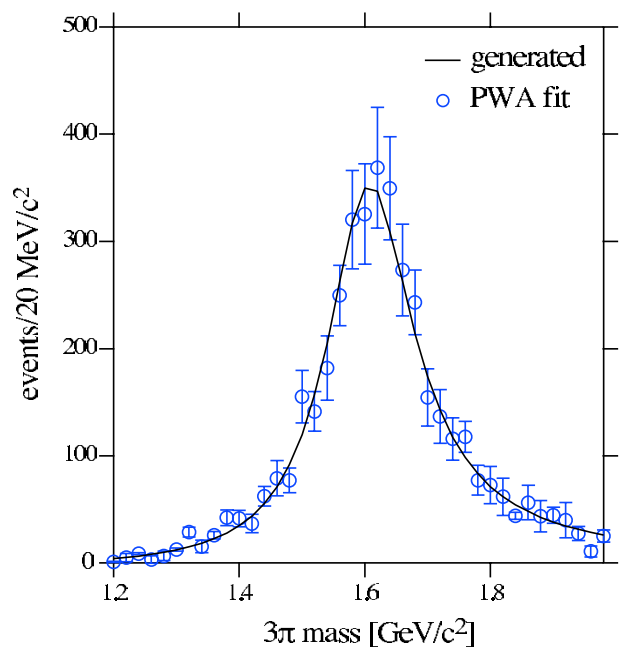

Figure 3. The results of a doubleblind Monte Carlo exercise showing the $J^{P C}=1^{-+}$exotic wave after fitting (open circles) and the exotic wave input (curve) into the mix of $\gamma p \rightarrow$ $\pi^{+} \pi^{+} \pi^{-} n$ events that were generated in this study.

access this mass range, and are achievable starting with $12 \mathrm{GeV}$ electrons. The photon beam will be produced off a crystal radiator using the coherent bremsstrahlung technique. For a typical crystal orientation, the resulting photon energy spectrum after collimation produces a peak at $9 \mathrm{GeV}$. The average degree of linear polarization in the peak is 40 percent. In addition, the photons emitted in a $0.5 \mathrm{GeV}$-wide window will be tagged using a magnetic spectrometer to detect the corresponding electrons.

\subsection{GlueX performance}

The sensitivity of the experiment, GlueX, to map the hybrid spectrum is determined by the performance of the solenoidal-based detector [4] and the flux and linear polarization of the photon beam. A double-blind exercise was carried out in which an exotic signal, a $J^{P C}=1^{-+}$state with a mass of $1.6 \mathrm{GeV} / \mathrm{c}^{2}$ decaying into $\rho \pi$, was generated along with a mix of three well-established non-exotic states with masses of $1.2,1.3$ and $1.7 \mathrm{GeV} / \mathrm{c}^{2}$. In this exercise the exotic signal was generated at the level of $2.5 \%$ of the total sample. The momenta of the decay products of these particles were smeared according to the expected 


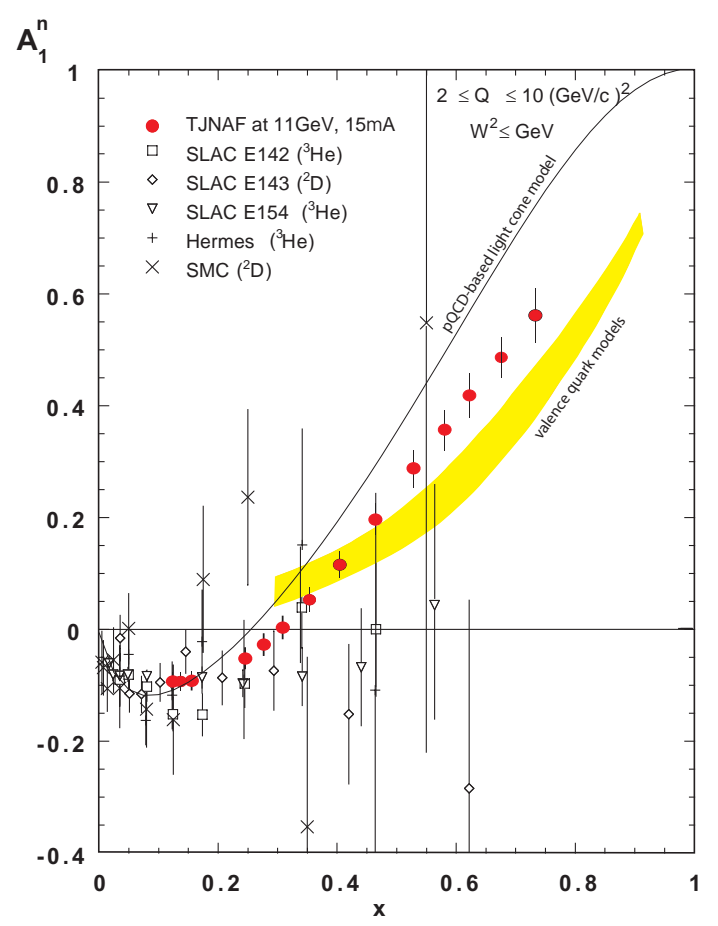

Figure 4. A projected $11 \mathrm{GeV}$ measurement of the neutron polarization asymmetry $A_{1}^{n}$ with a $15 \mu \mathrm{A}$ beam on a polarized ${ }^{3} \mathrm{He}$ target. The shaded band represents the range of predictions of valence quark models; the solid line is the prediction of a pQCD light-cone quark model.

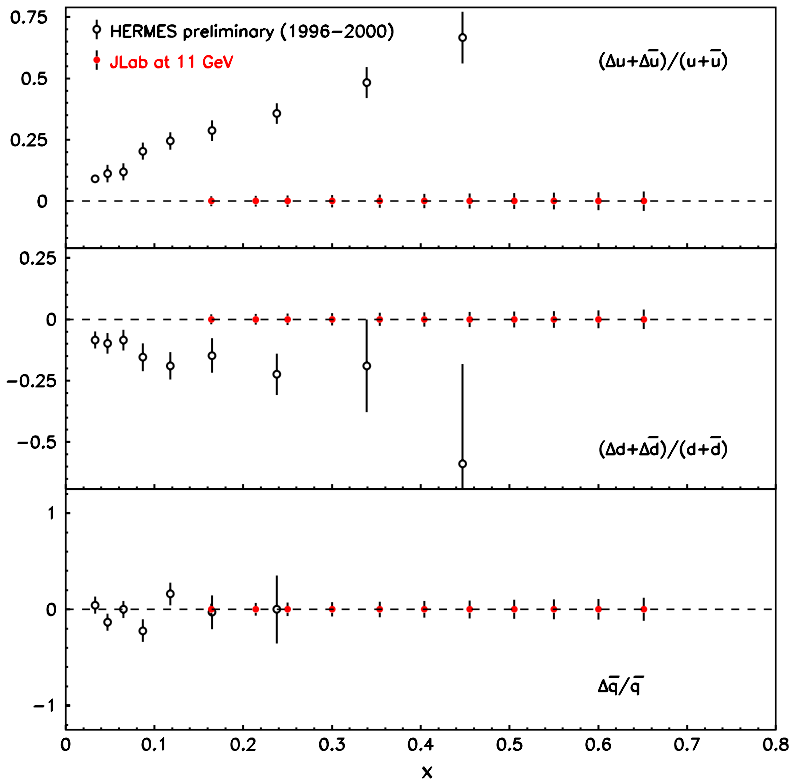

Figure 5. A semi-inclusive measurement of $\pi^{+}$and $\pi^{-}$production with polarized $\mathrm{NH}_{3}$ and ${ }^{3} \mathrm{He}$ targets allows a spin and flavor decomposition of the nucleon spin quark distributions. Factorization is assumed but will be tested and quantified by several additional measurements.

resolution of the detector. The acceptance of the detector was also applied. The resulting data set was passed through a partial wave analysis (PWA) fitting procedure to determine the relative contributions of each wave. Figure 3 shows the input exotic wave as a solid curve and the result of the PWA fit as data points with error bars. The input wave is reproduced extremely well, and this demonstrates the high sensitivity of the experiment to gluonic excitations.

\section{MOMENTUM DISTRIBUTION OF THE VALENCE QUARKS}

Deep inelastic scattering (DIS) experiments have led to the experimental confirmation of the existence of quarks, and to precision tests of QCD. However, there has never been an experimental facility with adequate duty factor and sufficient luminosity to measure DIS cross sections, especially polarized structure functions on the neutron, in the kinematic regime where the three basic ("valence") quarks of the nucleon dominate the wavefunction, i.e. for values of $x_{B j} \geq 0.5$. The $12 \mathrm{GeV}$ upgrade will allow us to map out the quark 


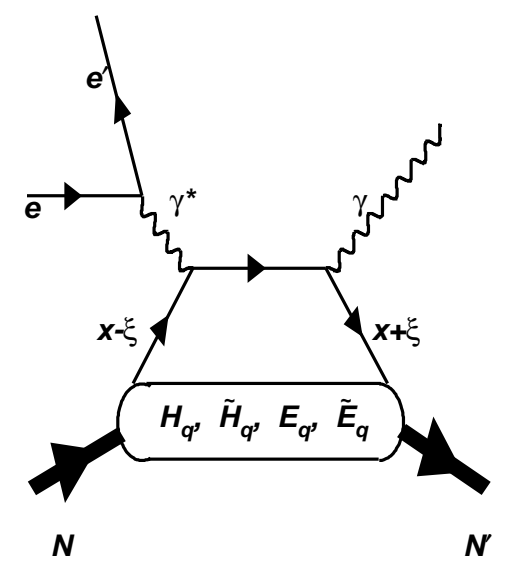

a)

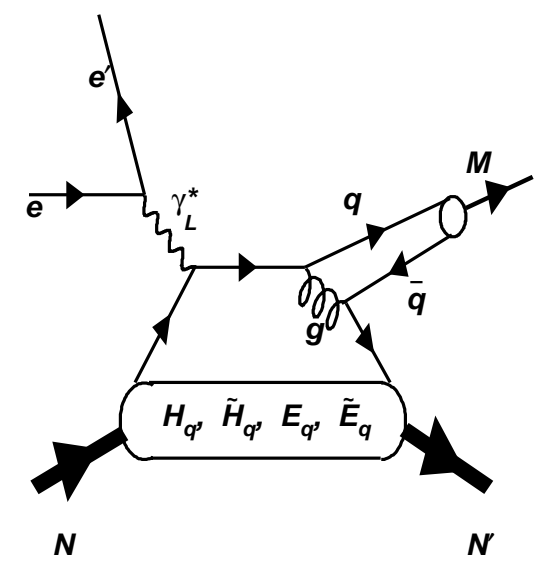

b)

Figure 6. a) Deep virtual Compton scattering (DVCS) and b) Deep virtual meson production.

distribution functions in this region with high precision.

Figure 4 shows one example of a measurement that is particularly well suited for the proposed upgrade. The neutron polarization asymmetry $A_{1}^{n}$ is sensitive to the spin wavefunction of the quarks. At large momentum transfers, the asymmetry is expected to approach one as $x \rightarrow 1$. This would be true in the valence region if the struck quark, which carries most of the nucleon momentum, also carries all of its spin. Existing data on $A_{1}^{n}$ do not extend to the region of valence quark dominance, and show no sign of making the predicted transition $A_{1}^{n} \rightarrow 1$. However, a dramatic improvement can be achieved in measurements of the neutron asymmetry using the $11 \mathrm{GeV}$ polarized beam and a polarized ${ }^{3}$ He target as shown in Fig. 4.

Unpolarized semi-inclusive meson production provides a means of extracting spin averaged quark and anti-quark distributions in the nucleon, and semi-inclusive production with a polarized beam on a polarized target offers the prospect of determining the spindependence of the individual quark species. After having shown the validity of factorization, these measurements can be used to determine the spin-dependent distributions $\Delta q\left(x_{B j}\right)$ and $\Delta \bar{q}\left(x_{B j}\right)$. Coincident measurements of $\pi^{+}$and $\pi^{-}$mesons from proton and neutron targets allow the flavor decomposition at $11 \mathrm{GeV}$ as shown in Fig. 5. Clearly these measurements will make a significant contribution to our understanding of the distribution of quark and anti-quark spins in the nucleon.

\section{3-D VIEW OF THE NUCLEON}

The information that can be obtained in DIS is limited to the square of the quark momentum wavefunction averaged over momenta transverse to the virtual photon direction. The formalism of the Generalized Parton Distributions (GPD's) has made it possible, in principle, to map out the complete wavefunctions. The GPD's are sensitive to the wavefunction at the amplitude level, instead of merely at the probability level, and, in particular, allow us to explore quark-quark correlations. They can be extracted from the cross sections for deep exclusive scattering (DES) processes with either a photon (Deeply 


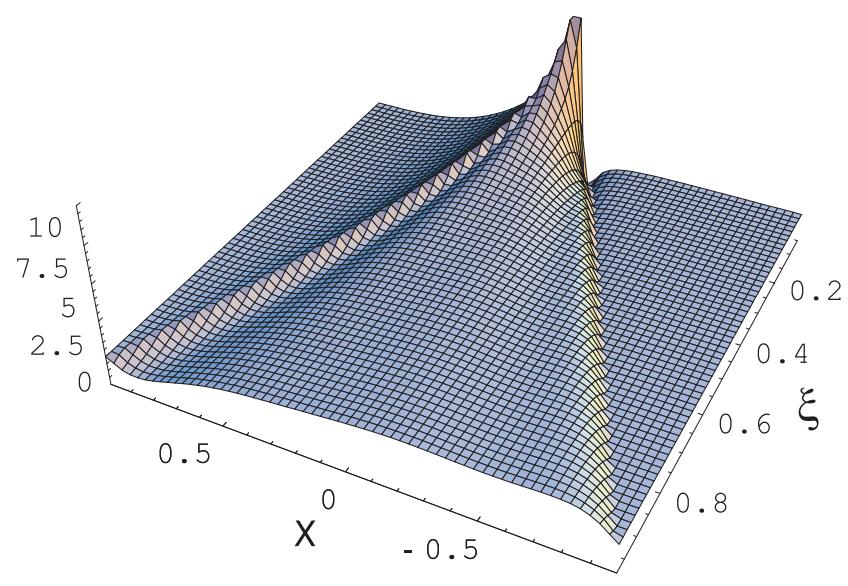

Figure 7. Model representation of the GPD $H(x, \xi, t=0)$ in two dimensions. The known parton momentum densities constrain the distribution at $\xi=0$. The new physics is contained in the $\xi$ dependence and the $t$ dependence of this surface, which can currently only be modeled. As $\xi$ increases the correlations between the quarks and antiquarks increase leading to meson-like distributions at large $\xi$.

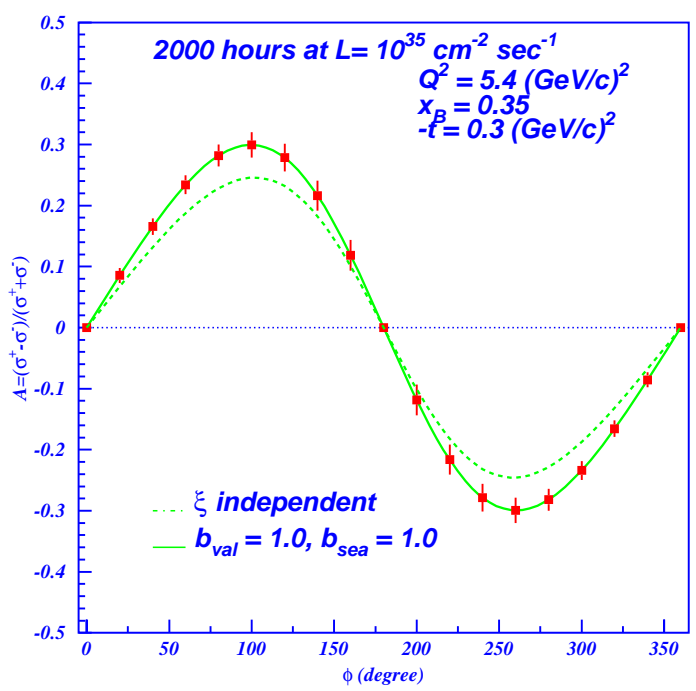

Figure 8. A projected measurement of the single-spin asymmetry for $e p \rightarrow e p \gamma$ with longitudinally polarized $11 \mathrm{GeV}$ electrons and a $Q^{2}=5.4$ $(\mathrm{GeV} / \mathrm{c})^{2}$. The curves indicate various GPD models, all of which are compatible with the known longitudinal parton momentum distributions.

Virtual Compton Scattering, DVCS) or a meson in the final state (Fig. 6). These processes access a rich body of new information about the full wavefunction, including non-forward overlaps of their longitudinal parts and their transverse momentum structure.

There are four GPD functions $(\mathrm{H}, \tilde{\mathrm{H}}, \mathrm{E}$, and $\tilde{\mathrm{E}})$ which are each functions of the longitudinal momentum fraction $x$, the longitudinal momentum transfer $\xi$, and the four-momentum transfer t. The new physics which can be accessed using DES reactions is contained in the dependence on $\xi$ and $t$. The model dependence on the longitudinal momentum transfer $\xi$ is illustrated in Fig.7. The $\mathrm{t}$ dependence is directly related to the distribution of the parton densities as a function of impact parameter $\left(b_{\perp}\right)$, thus providing 3-dimensional images of the nucleon. The Fourier transforms of $\mathrm{H}$ and $\tilde{\mathrm{H}}$ can be interpreted as parton distribution functions which give the longitudinal momentum distribution of quarks as a function of their transverse position $b_{\perp}$. The transform of $H$ is given by the spin averaged structure of the nucleon, whereas $\tilde{H}$ reveals the spin structure of the nucleon as a function of $x$ and $\mathrm{b}_{\perp}$. The physical picture of $\mathrm{E}$ and $\tilde{\mathrm{E}}$ is a subject of active research, but correspond to nucleon helicity flip amplitudes.

The complete picture of the nucleon can only be revealed by an extensive program dedicated to measuring a range of exclusive reactions. The quantum numbers of particles in the final state can be used as filters to select specific combinations of the four GPD functions. For example, DVCS is sensitive to all four $\mathrm{H}, \tilde{\mathrm{H}}, \mathrm{E}$, and $\tilde{\mathrm{E}}$. Vector meson production is sensitive to $\mathrm{H}$ and $\mathrm{E}$, and pseudoscalar meson production selects out the 
functions $\tilde{\mathrm{H}}$ and $\tilde{\mathrm{E}}$. Polarization observables, both beam and target, are sensitive to interference terms, so they will be extremely valuable to help sort out various contributions to the nucleon structure.

There are two general experimental challenges to extract the GPD's from DES measurements. Firstly, cross section measurements represent integrals over $x$, and unfolding the GPD functions from the data, even under ideal conditions, is a complex mathematical exercise. Secondly, the kinematic range over which measurements must be done to ensure that DES scaling is applicable needs to be determined experimentally. The necessary condition is that the underlying processes can be factorized into a "hard" pQCD scattering amplitude and a "soft" amplitude which arises from the quark wavefunctions. The initial emphasis of the program will be DVCS, since scaling is expected to be reached early in this case. For meson production, an additional hard collision is required which will likely shift the onset of scaling to higher momentum transfers.

As an example for what can be achieved experimentally using the upgraded large acceptance detector CLAS in Hall B at a luminosity of $L=10^{35} \mathrm{~cm}^{-2} \mathrm{~s}^{-1}$ is shown in Figure 8. The single-spin asymmetries for DVCS at $Q^{2}=5.4(\mathrm{GeV} / c)^{2}$ for a 2000 hour run will provide sensitive constraints for the GPD's.

\section{SUMMARY}

We have presented the physics program which motivates the JLab accelerator upgrade to $12 \mathrm{GeV}$. The unique combination of high energy, high duty-cycle, high intensity and small phase space beams from CEBAF's superconducting accelerator, in combination with complementary equipment in four experimental halls, will allow unprecedented precision in the measurement of the structure of mesons, nucleons, and nuclei.

\section{REFERENCES}

1. K. de Jager, contribution to this conference.

2. J. Symons, et.al., "Opportunities in Nuclear Science, A Long-Range Plan for the Next Decade," April 2002. Available at http://www.nscl.msu.edu/future/lrp2002.html.

3. L.S. Cardman et al., "White Paper: The Science Driving the $12 \mathrm{GeV}$ Upgrade of CEBAF," 2001. Additional details can be found at http://www.jlab.org/div_dept/physics_division/GeV.html.

4. The GlueX/Hall D Design Report, November 2002. Additional details and references can be found at http://www.gluex.org.

5. The Hall A Preconceptual Design Report, November 2002. Additional details and references can be found at http://hallaweb.jlab.org/12gev.

6. The Hall B Preconceptual Design Report, November 2002. Additional details and references can be found at http://clasweb.jlab.org/12gev.

7. The Hall C Preconceptual Design Report, November 2002. Additional details and references can be found at http://www.jlab.org/Hall-C/upgrade. 